\title{
Structural modelling and testing of failed high energy pipe runs : 2D and 3D Pipe Whip
}

\author{
S.R. Reid ${ }^{1}$, B. Wang ${ }^{2}$ and M. Aleyassin ${ }^{1}$ \\ ${ }^{1}$ Schoolof Engineering, University of Aberdeen, Aberdeen AB24 3UE \\ ${ }^{2}$ School of Engineering and Design, Brunel University, Uxbridge UB8 3PH
}

\begin{abstract}
The sudden rupture of a high energy piping system is a safety-related issue and has been the subject of extensive study and discussed in several industrial reports [e.g. 2-4]. The dynamic plastic response of the deforming pipe segment under the blow-down force of the escaping liquid is termed pipe whip. Because of the potential damage that such an event could cause, various geometric and kinematic features of this phenomenon have been modelled from the point of view of dynamic structural plasticity. After a comprehensive summary of the behaviour of in-plane deformation of pipe runs $[9,10]$ that deform in $2 \mathrm{D}$ in a plane, the more complicated case of 3D out-of-plane deformation is discussed. Both experimental studies and modelling using analytical and FE methods have been carried out and they show that, for a good estimate of the "hazard zone" when unconstrained pipe whip motion could occur, a large displacement analysis is essential. The classical, rigid plastic, small deflection analysis (e.g. see [2 and 8]), is valid for estimating the initial failure mechanisms, however it is insufficient for describing the details and consequences of large deflection behaviour.
\end{abstract}

Keywords: Pipe whip, dynamic structural plasticity, safety design, large plastic deformation 


\section{Introduction}

The design and construction of nuclear power plants for 'new build' projects have changed significantly over the years. There is an increased use of and reliance on computer-based plant design management systems that can integrate design, CAD manipulation, structural integrity assessments, manufacture, construction and safety considerations [1].

One of the un-met reqirements is to enhance the capability of the plant design management systems to give ‘safe by design’ capability for high energy piping systems. In a typical nuclear power plant, the sudden rupture of a high energy piping system is a safety-related issue. A sudden rupture results in a force from the escaping fluid (jet impingement or blow-down force) and dynamic motion of the pipe, i.e. pipe whip. Designers have to ensure that any safety-related equipment is either protected or is out of the Hazard Zone where a whipping pipe may strike. In the past, the R3 Impact Assessment Procedure [2, 3] developed in the UK nuclear industry and the American code ANSI/ANS 58.2 [4] have been used to assess pipe rupture hazards. Particularly with regard to the structural deformation and motion of the pipe, which are responsible for some of the potential damage (note: the variations in the flow, fluid-structure interaction and jet loading is not an issue to be dealt with here), these procedures now need to be updated. More recent pipe whip mechanics research, and its application needs to be added to the capability in the commercial codes, such as PDMS [1], being used by designers.

As far as structural modelling is concerned, many of the published models [e.g. 5 - 7] are based on small-deflection, rigid, perfectly-plastic approaches [2, 8], focusing on the initial failure mechanisms identifying both the number and the location of the plastic hinges. The consequent results are valid when the motion of the pipe segment is limited. They provide good guidance for the design and positioning of pipe-run supports and restraints. However, for unconstrained pipes, where both the 
deformation and deflection can be large following a rupture, the dynamic response of the whipping pipe is potentially a more serious safety concern. Such events need to be predicted by an improved modelling capacity, tested for verification, and, eventually, be made available through a 3D virtual model of the plant, to help safeguard the workplace and any safety critical equipment.

In this paper, first an experimental study of 2D in-plane and 3D out-of-plane of high-energy pipe runs performed in UMIST in 1996-2000 is outlined. Next, the 2D bending-dominated behaviour of pipe segments undergoing motion in a plane is discussed. This includes an account of modelling developments using finite element analysis and other techniques showing predictions of the Hazard Zone and the time history of the dynamic plastic behaviour of the deforming pipe. Finally the 3D modelling of pipe whip involving combined bending and torsional deformation is presented.

\section{Experiments}

Pipe whip tests were carried out on the pneumatic facilities designed and built originally at UMIST. The system consists of a high pressure reservoir, the test pipe and the rupture device which allows the suddenly release of high pressure fluid (air). Details of the facility, test procedures and measurement are sketched in Fig. 1a and described in [9].

The test pipes had various geometries, some including restraints. However here emphasis will be on freely whipping straight cantilever pipe specimens and pipes with right-angle bends loaded in their plane and out-of plane. At the end of the pipe a cold-formed $90^{\circ}$ elbow was attached in the appropriate direction to effect the desired loading. A flange was brazed on to the end with a Melanex(C) sheet to seal the pipe. This allowed the pressure to be built up in the pipe which was connected to a pre-pressurised reservoir. The whipping process was triggered by rupture the Melanex $\bigodot$ sheet using a simple 'fuse wire' device, allowing jetting of the working fluid (air) to create the blowdown force pulse. The volume of the reservoir was designed to be sufficient to allow no more than a 
roughly $10 \%$ pressure drop at the completion of the test. The blow-down force produced by the jetting is a follower-force with the direction maintained perpendicular to the centroidal axis of the adjacent pipe. Pressure transducers P1, P2 and P3 were placed at key locations to record pressure changes. After allowing the pipe to deform until the final mode (root rotation) was attained, the pipe was arrested by its striking a barrier, shown schematically in Fig. 1a.

Large deformation and dynamic motion of the pipe lead to acceleration of the pipe, plastic deformation, reduction of the cross section of the pipe (ovalisation or collapse) at the kink locations which limits the flow, thus reducing the pressure at the outlet. These factors are the main structural concerns of this paper. In order to estimate the variation of the pressures, certain 'constrained' tests were performed to ensure that good estimates for the blow-down forces in particular tests and for particular pipee geometries were obtained for modelling purposes. This is described in [9]. Fig. 1a shows the test setup, two sets of typical pressure measurements are shown in Fig. 1b on straight and bent pipes, and film clips from high speed photography are shown in Figs. 1c, 5, 7, 8 and 9. Interestingly, all the pipes loaded in the plane of the pipe remained within the plane in which they first moved, demonstrating the stability of this motion. This behaviour is treated in Section 3. Out-ofplane loading introduced torsional motions and resulted in the generation of a 3D Hazard Zone and this work is treated in Section 4.

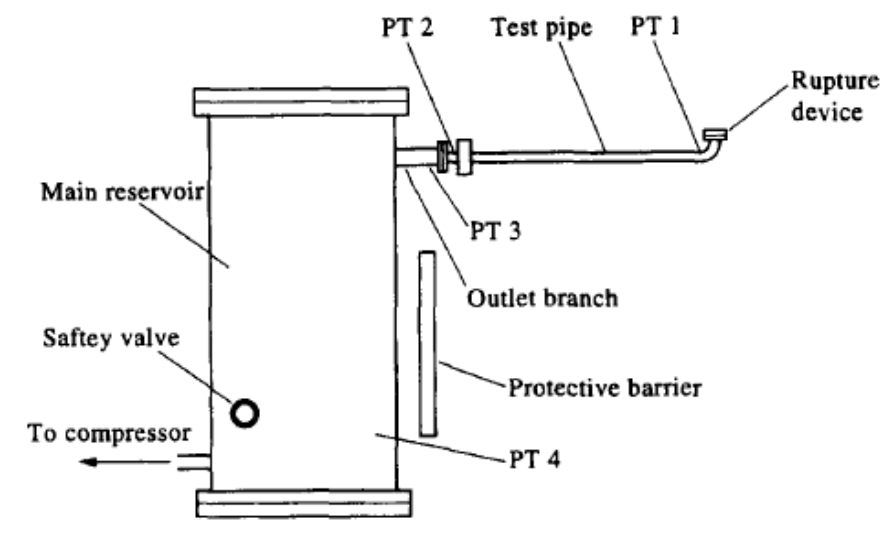


Fig. 1a Experimental set-up [9]

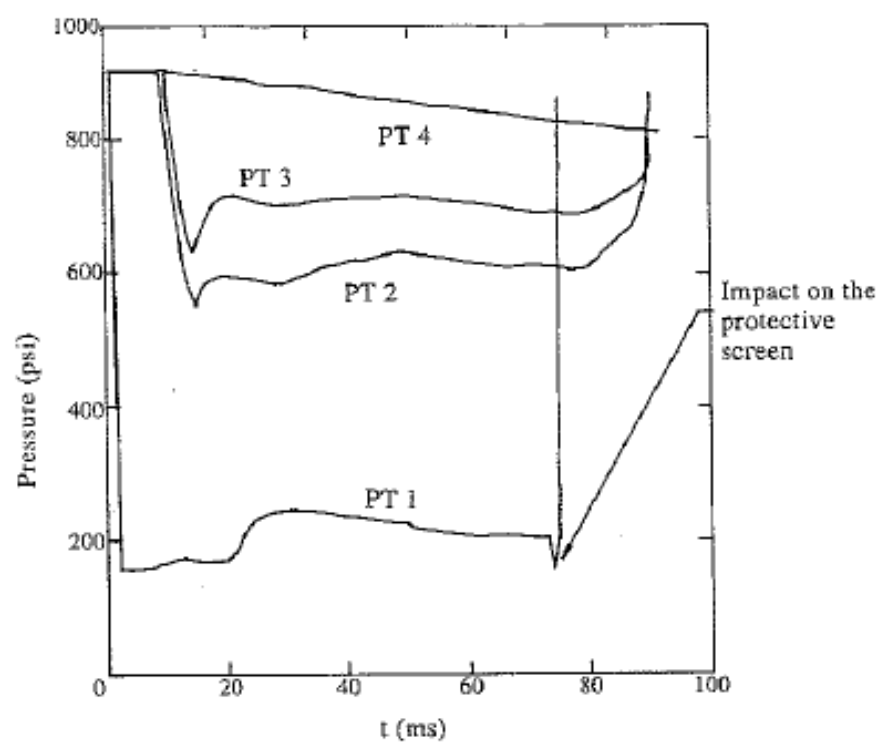

(i)

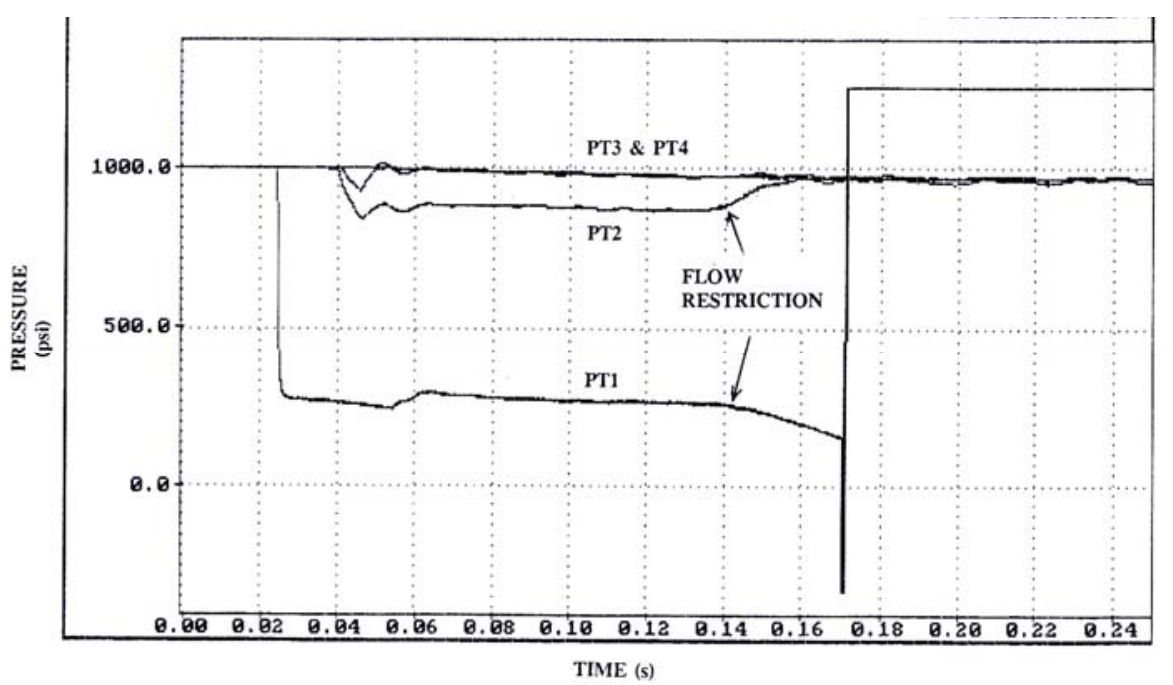

(ii)

Fig. 1b (i) Test 4 - cantilever straight pipe - pressure transducer traces

(ii) Test 41 - bent pipe loaded out-of-plane - pressure transducer traces 


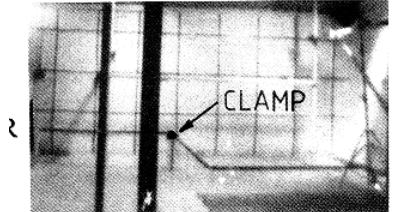

(i) $t=0 \mathrm{~ms}$

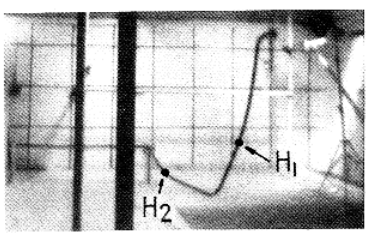

(iii) $t=35 \mathrm{~ms}$

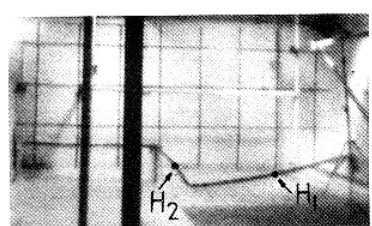

(ii) $t=10 \mathrm{~ms}$

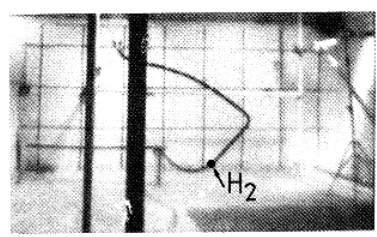

(iv) $t=50 \mathrm{~ms}$

Fig. 1c Clip from high speed photography (1000f/s), in this case with the initial locations of internal 'hinges' $H_{1}$ and $H_{2}$ formed in the early stages of plastic deformation.

\section{Bending-only (2D) pipe whip}

\subsection{Small displacement, rigid perfectly-plastic model}

There is an extensive literature on in-plane pipe whip analysis of straight pipes based on elastic (or rigid)-plastic cantilever beam models. A selection of such models is cited in [10]. A small deflection analysis of a straight cantilever pipe subjected to a force pulse see e.g. [5, 11 appendix 1] shows that, above a certain blow-down force, a stationary-plastic hinge is formed in the pipe, Fig. 2(a) as shown in Fig. 2(b)

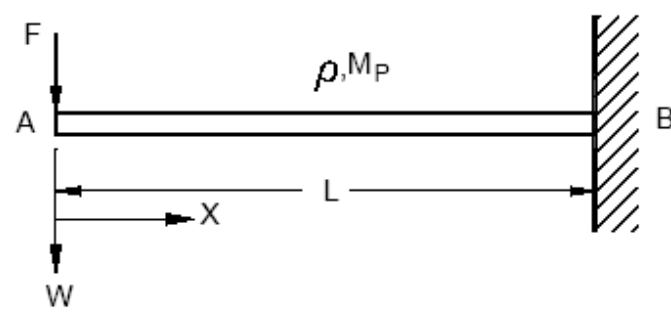

(a)

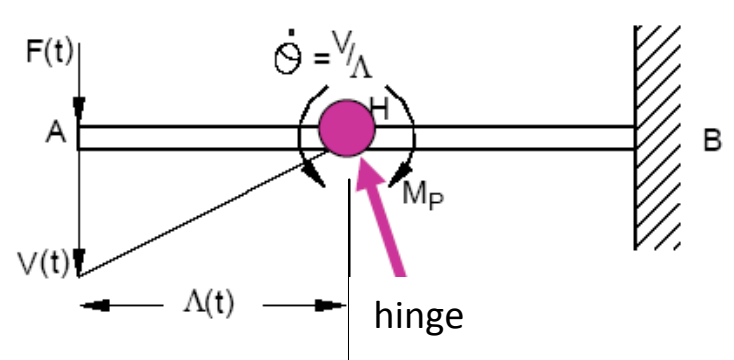

(b)

Fig. 2 A cantilever pipe subjected to a suddenly applied force pulse at the end. $\dot{\theta}$ is the angular velocity at the plastic hinge. 
The initial position of the plastic hinge is related to the magnitude of the applied force, the plastic bending strength, $M_{P}$ (for a pipe of diameter $D$, wall thickness $t$ with a yield stress $\sigma_{Y}$ ). $M_{p}=D^{2} t \sigma_{Y}$ is the plastic bending moment of the pipe cross-section. If the tip mass is zero (account can be taken for any concentrated masses, e.g. flanges, at the tip) and for a constant impact force $F$, a simple analysis shows that initially three deformation modes could appear, governed by the magnitude of $F$ :

$$
\begin{array}{ll}
0<F<\frac{M_{P}}{L} & \text { no motion } \\
\frac{M_{P}}{L} \leq F<\frac{3 M_{P}}{L} & \text { hinge at root } \\
\frac{3 M_{P}}{L} \leq F & \text { hinge within pipe - see Fig. 2b }
\end{array}
$$

where $L$ the length of the pipe. For the third mode, the plastic hinge appears at

$$
x_{0}=\frac{3 M_{P}}{F},
$$

measured from the tip. For a constant force, the small deflection analysis leads to a stationary hinge. For a reducing force, the hinge travels towards the root of the cantilever. The instantaneous position of the hinge is given by

$$
\Lambda(t)=\frac{3 M_{P} t}{\int_{0}^{t} F(\tau) d \tau},
$$

measured from the loading point. When the travelling hinge reaches the pipe root, it becomes a root hinge (the so-called modal solution [11]). For a finite input energy, the pipe eventually stops rotating about its root when the remaining kinetic energy is dissipated in plastic deformation. As a rough guide, the ratios of the energy dissipated during each of the stages, i.e. stationary hinge, travelling hinge and root rotation, can be calculated [12]. The energy dissipated during the three-phase response (stationary hinge, $E_{s}$ travelling hinge $E_{t}$ and root rotation $E_{r}$ ) under a rectangular pulse can easily be shown to be as a proportion of the input energy $E_{\text {in }}$, 
$\frac{E_{s}}{E_{\text {in }}}: \frac{E_{t}}{E_{\text {in }}}: \frac{E_{r}}{E_{\text {in }}}=\frac{1}{3}: \frac{2}{3}\left(1-3 \frac{M_{P}}{F L}\right): \frac{2 M_{P}}{F L}$

where

$E_{\text {in }}=\frac{1}{3} \frac{F^{3} L^{3} \tau^{2}}{M_{P}^{3}}$

$F$ and $\tau$ being the magnitude and duration of the rectangular force pulse.

The small deflection, rigid perfectly-plastic (r-p-p) model provides a simple method to identify the location of initial hinges, allowing restraints to be positioned to prevent the progress of the whipping motion of the pipe. Equation (3) gives an interesting insight into the significance of travelling hinges as an energy absorbing mechanism, depending as it does on the magnitude of the applied force pulse. However, free motion could pose a significant safety hazard from a broken high energy pipe-run. In the subsequent sections, 2D and 3D layouts are discussed using large deflection analysis to provide more accurate estimates of the deformed (or deforming) profile of pipe segments.

\subsection{Large displacement bending behaviour}

\subsubsection{Equations of motion}

A large deflection model, essentially a beam model, has been described in a number of works, e.g. $[9,10]$. The governing equations are given in full here as they also apply to other techniques, e.g. Section 3.4.1.2. First, the large deflection features were introduced using the equations governing the dynamic motion of the pipe sections, as illustrated in Fig. 3. The coupled equations of motion for the Cartesian axial and transverse displacement components along the centroidal axis of the pipe, $u(s, t)$ and $w(s, t)$ are given by:

$$
m \ddot{u}=\frac{\partial}{\partial s}(N \cos \theta)+\frac{\partial}{\partial s}(Q \sin \theta)+p_{x}
$$




$$
m \ddot{w}=\frac{\partial}{\partial s}(N \sin \theta)-\frac{\partial}{\partial s}(Q \cos \theta)+p_{y}
$$

where $m$ is the mass per unit length, $p_{x}(s, t)$ and $p_{y}(s, t)$ are spatially and temporally varying external applied force densities along the two Cartesian co-ordinate directions (usually reduced to a single follower force pulse $\bar{Q}(t)$ at the tip), and the dots denote time derivatives. $\theta(s, t)$ is the angle of the deformed beam centreline with respect to the positive $X$-direction. The spatial co-ordinate variables $\mathrm{x}$ and $\mathrm{s}$ are related by,

$$
\frac{d s}{d x}=\sqrt{\left(w^{\prime}\right)^{2}+\left(1+u^{\prime}\right)^{2}}=q(x)
$$

where $w^{\prime}=d w / d x$ and $u^{\prime}=d u / d x$. The quantity $\theta$, is

$$
\theta=\tan ^{-1}\left(\frac{w^{\prime}}{1+u^{\prime}}\right)
$$

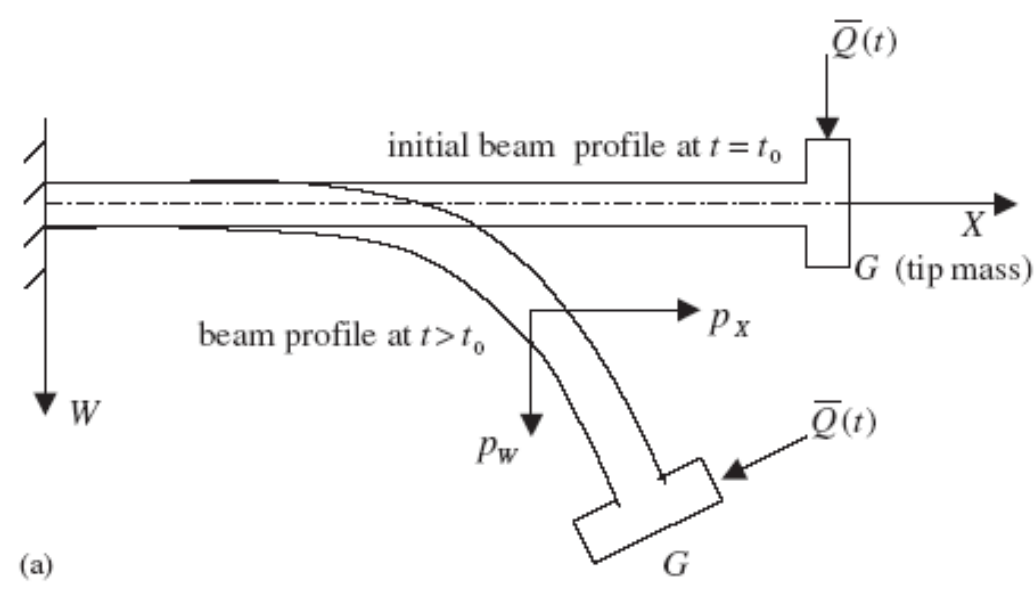




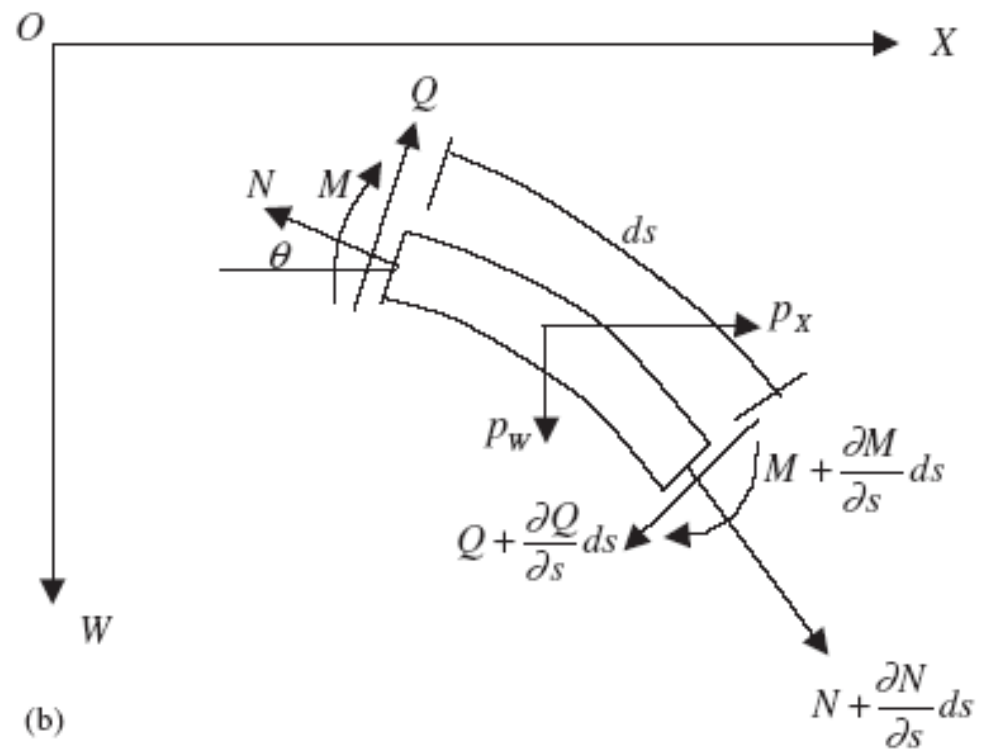

Figure 3. (a) A cantilever beam with a lumped end mass, G, loaded by a follower shear force $\bar{Q}(t)$ at the free end; and (b) an infinitesimal beam element in the deformed configuration.

The axial strain, $\varepsilon_{0}$, and the curvature, $\kappa$, of the centreline of the deformed beam element can be expressed in terms of $u$ and $w$ by

$$
\begin{aligned}
& \varepsilon_{0}=\frac{d s-d x}{d x}=q(x)-1 \\
& \kappa=\frac{d \theta}{d s}=\frac{\left(1+u^{\prime}\right) w^{\prime \prime}-w^{\prime} u^{\prime \prime}}{q^{3}}
\end{aligned}
$$

\subsubsection{Constitutive model}

Associated with the large deformation described above, an Euler-Bernoulli beam theory was used as described and discussed in [10]. An elastic-plastic hardening-softening (e-p-h-s) bending relationship is required to describe the nonlinearity in material hardening and structural softening. The latter is due to the ovalization and possibly collapse, forming a 'kink', at a particular cross-section. It 
is assumed in the model that the non-linear $M-\kappa$ relations remain unaffected by changes in the axial force $N$, even in the softening range. While this is done for purposes of simplicity, it turns out to be a reasonable approximation for tubular beams wherein the maximum absolute value of $N$ remains very much below its elastic limit $N_{e}$. thus the e-p-h-s $M-\kappa$ relations takes the general form:

$$
M=F(\kappa)= \begin{cases}E I \kappa & \kappa<\kappa_{e} \\ M_{e}+A\left(\kappa-\kappa_{e}\right)-B\left(\kappa-\kappa_{e}\right)^{2} & \kappa_{e}<\kappa<\kappa_{c r}, \Delta \kappa>0 \\ M_{\max }\left\{1-\alpha\left(\kappa-\kappa_{c r}\right)^{2}\right. & \kappa_{c r}<\kappa<\kappa_{c o l}, \Delta \kappa>0 .\end{cases}
$$

as illustrated in Figure 4, corresponding to initial elastic deformation, followed by a plastic hardening stage, then by a plastic softening (due to the change on cross-sectional geometry) phase. Beyond that, the $M-\kappa$ relation reduces to a flat line parallel to the $\kappa$ - axis and this corresponds to the residual moment carrying capacity of a collapsed beam section. $A$ and $B$ are constants, deduced from the boundary condition.

$$
A=\frac{2\left(M_{\max }-M_{e}\right)}{\kappa_{c r}-\kappa_{e}} \text { and } B=\frac{M_{\max }-M_{e}}{\left(\kappa_{c r}-\kappa_{e}\right)^{2}} .
$$

The softening parameter, $\alpha$, affects the local slope of the softening branch of the $M-\kappa$ curve and is thus responsible for determining the post-peak-load moment carrying capacity of the beam section. The thinner the pipe, the higher is $\alpha$ and the steeper is the fall of the softening branch. 


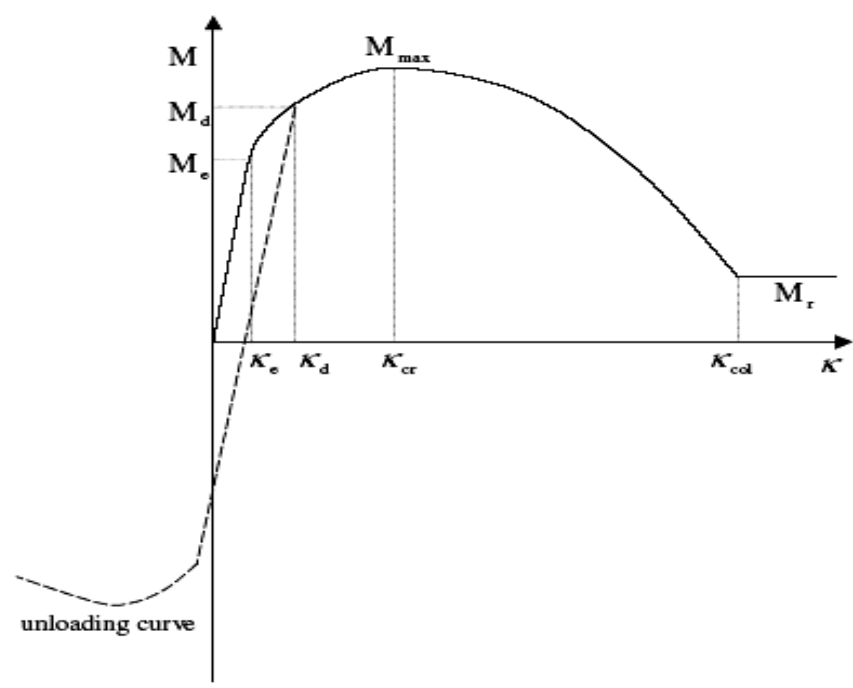

Figure 4. A typical elastic-plastic hardening-softening (e-p-h-s) moment-curvature relationship following Reid et al. [10]

The discussion above is valid only for the loading states, i.e. $\dot{\kappa}>0$. The elastic unloading curve is considered approximately to be parallel to the loading one and the reverse yielding follows the dashed line (as indicated in Fig. 4). Thus, assuming isotropic plastic behaviour, the $M-\kappa$ relations during unloading takes the form:

$$
M=F(\kappa)= \begin{cases}M_{d}+E I\left(\kappa-\kappa_{d}\right) & \kappa<\kappa_{e} \\ -f_{h}\left(\kappa_{d}+\left|\kappa-\kappa_{e}\right|\right) & \kappa_{e}<\kappa<\kappa_{c r}, \Delta \kappa>0 \\ -f_{s}\left(\kappa_{d}+\left|\kappa-\kappa_{e}\right|\right) & \kappa_{c r}<\kappa<\kappa_{c o l}, \Delta \kappa>0 .\end{cases}
$$

$\kappa_{d}$ and $M_{d}$ are respectively the curvature and bending moment of the beam section at the instant when unloading is initiated, and $\kappa_{g}=\kappa_{d}-2 M_{d} / E I$ is the reverse yielding curvature. The curvature at collapse, $\kappa_{\text {col }}$ may be obtained from the equation of the softening branch as $\kappa_{\text {col }}=\sqrt{\frac{M_{\max }-M_{r}}{\alpha M_{\max }}}$ 
The values of the key parameter for a particular pipe can be deduced either experimentally or theoretically as explained in [14], thus completing the governing equations.

\subsection{Numerical results}

\subsubsection{Straight pipe}

\subsubsection{EPHS model}

Figure 5 shows a comparison between the experimental observations and the e-p-h-s model $[9,10]$ and results from the finite element code DYNA 3D [14]. A full account of the experimental study was given in [9]. The shapes calculated by the central difference method $[9,10]$ and the finite element results using shell elements produced by DYNA 3D. The good agreement between the deformed profiles is evident. The numerical results allow a detailed picture of the dynamic elasto-plastic deformation development to be built along the pipe length as a function of time $[15,16]$.
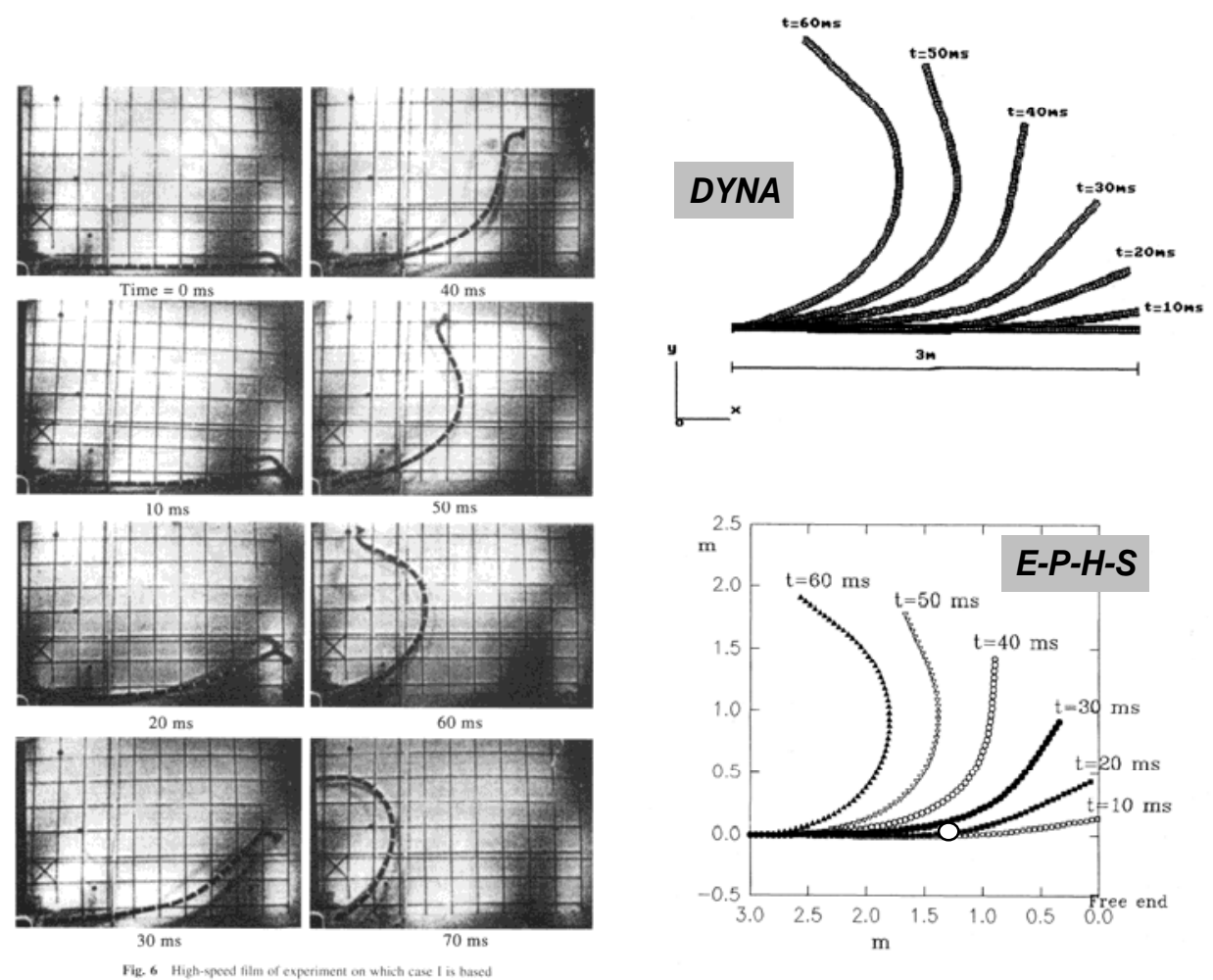
Figure 5. Comparison between experiments, e-p-h-s and finite element shell model from [15,16]. The circle in the E-P-H-S figure indicates the initial hinge position as predicted by the small deflection r-p-p model. .

\subsubsection{Mesh-free models}

The literature about whipping of the straight pipe is extensive and the governing equations (5)(14) for large deflections have been solved by central difference (CD) methods $[9,10]$ as described above, where is it shown [10] that the method sometimes produces numerical instability in the determination of pipe curvature. Later Reid and Roy [16] used mesh free method to tackle the numerical instability in the CD approach. The Gaussian Sinc Collocation (GSC) method [17] and the Reproducing Kernel Particle Method (RKPM) [18] have been used to refine the instability in the solution.

As an illustration, Fig. (6) shows that the irregular jumps in the curvature occur at times when the pipe faces structural softening. These jumps are not real, but are the result of a CD solution affected by softening. Fig. 6 also shows how RKPM produces more stable solutions and removes the 'jumps' in the curvature. 


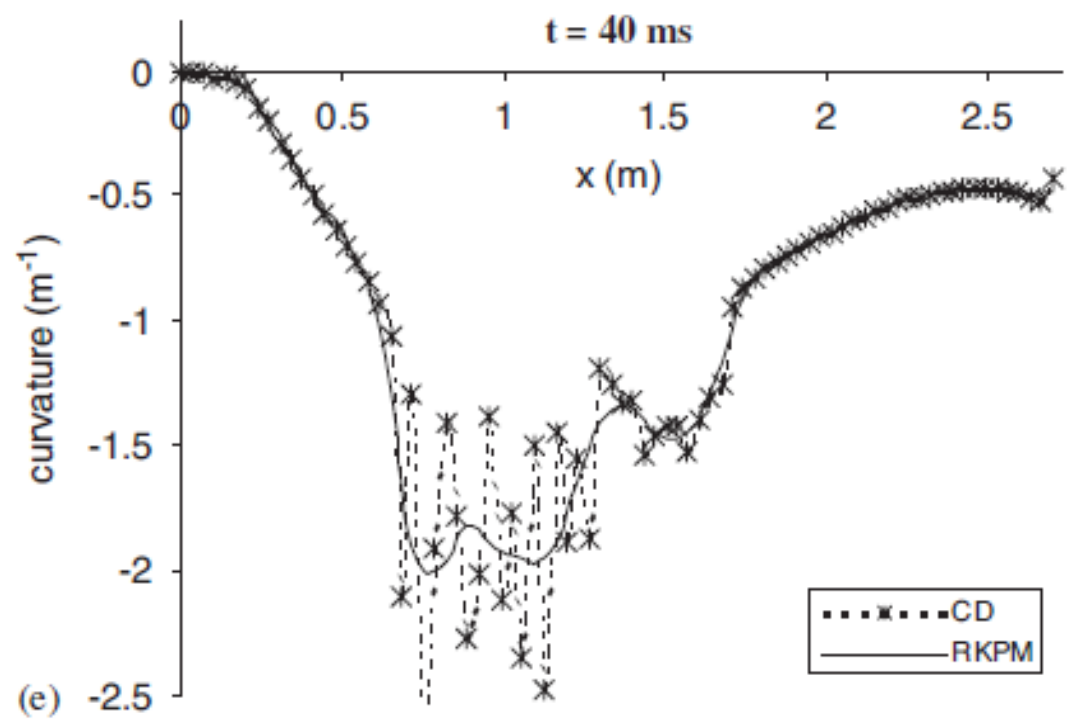

Figure 6: When $\alpha=0.001$ (see equation 11) and pipe after $t=40 \mathrm{~ms}$ faces structural softening CD method results in false curvature 'jumps' but RKPM removes these.

\subsubsection{In-plane loading of bent pipes: closing mode of right angled bent pipe}

For 2D pipe runs, when thrust force is applied in the plane of the bent pipe, the bend angle will open or close, depending on the direction of the thrust. Though the bend angle can be of any value as having been studied in [19], a right-angled bend is probably the most commonly used in piping runs, thus is discussed here.

The closing mode has also been modelled using the above e-p-h-s beam theory by Reid et al in [20] and again was not only shown to be in good agreement with the relevant experiments [9] but also verified by FEM [15]. The comparisons are shown in Figure 7.
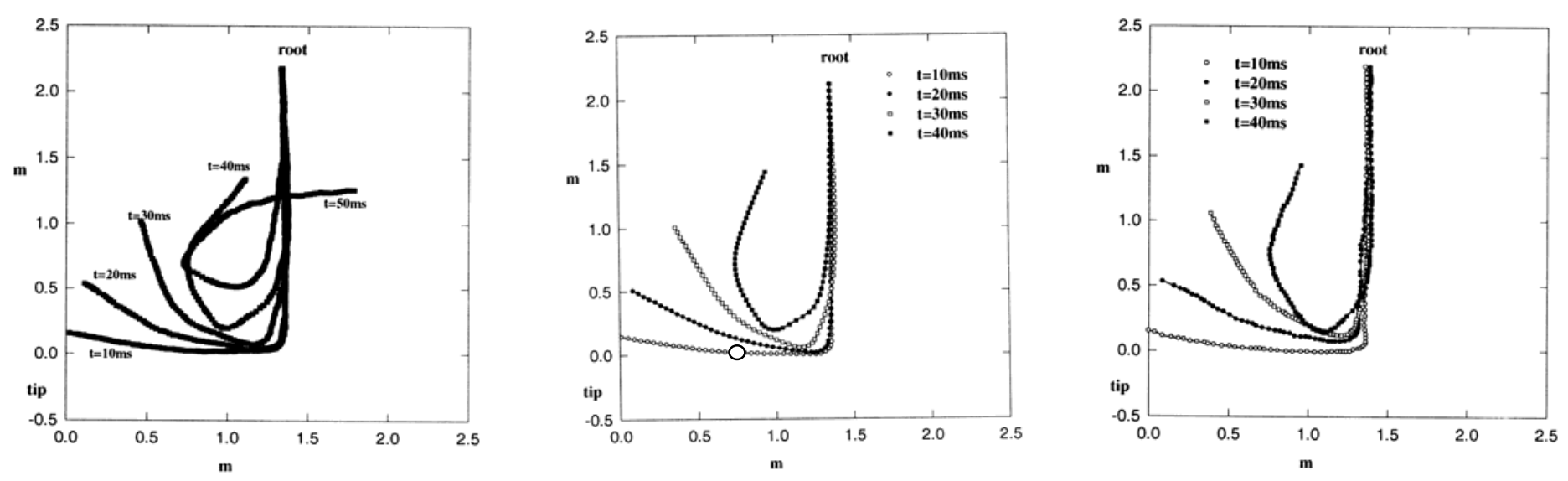
Figure 7. Comparison between DYNA3D, E-P-H-S and experiments models for the closing mode. The circle in the E-P-H-S figure indicates the initial hinge position as predicted by the r-p-p model [15, 16, 20].

\subsubsection{Opening mode of right angled bent pipe}

Similar to the above closing mode, the opening model of a 2D right angled pipe was modelled [20]. Results are given in Fig. 8 with experimental comparisons. A clear feature of the response is the rotation in the reverse sense of the bending caused by the follower force (see Fig. 7 from 30ms). This rotation is due to the elastic effect of the material of the pipe. Interestingly, such a "reverse" rotation is largely absent in the closing mode.

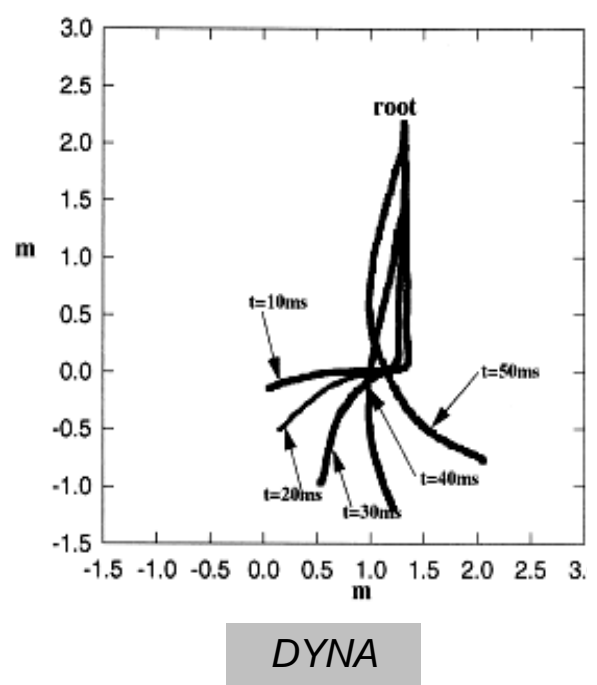

(a)
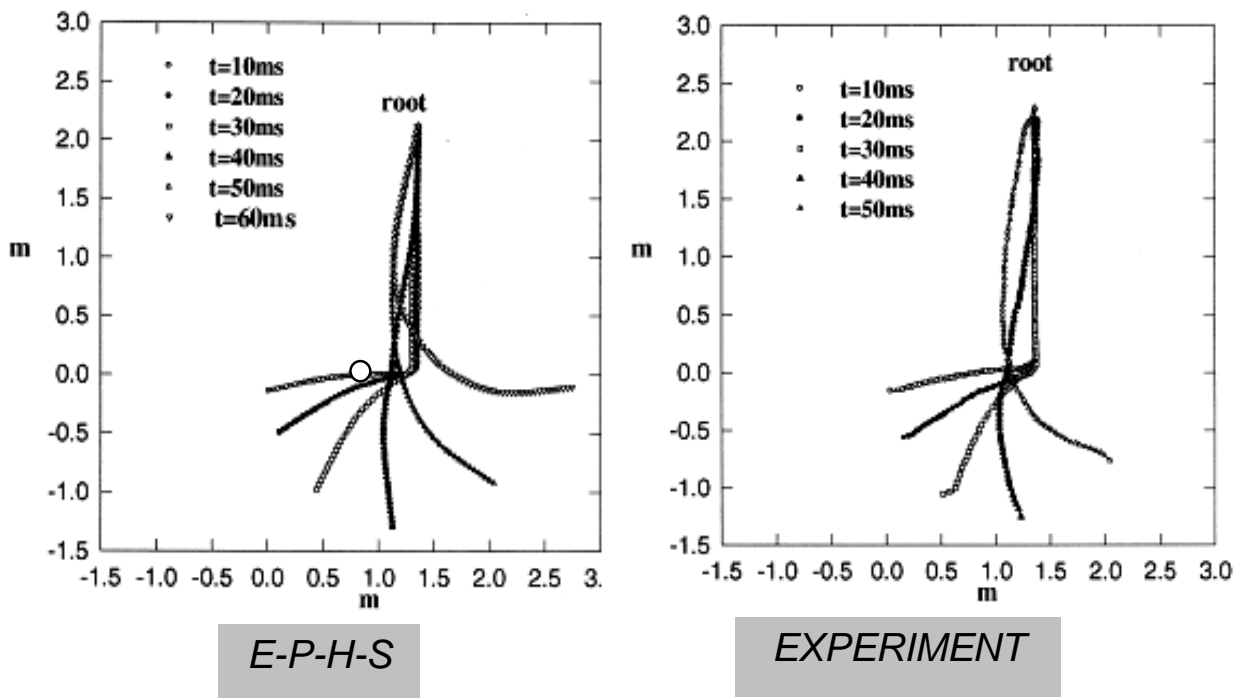

(b) 
Fig. 8 Comparison between DYNA3D, E-P-H-S models and experiment for the opening mode. The circle in the E-P-H-S figure indicates the initial hinge position as predicted by the r-p-p model

$[15,16,20]$.

\section{Out-of-plane (3D) pipe whip}

For out-of-plane pipe whip, the thrust force is normal to the plane of the bent pipe, thereby causing both bending and torsion in the pipe. Thus, the torsional deformation, provides dynamic motion in the pipe which could be particularly volatile and hazardous. The geometric complexity in the free whipping motion of a dynamically deforming pipe, which involves both bending and torsion involving large deflections and rotations in 3D space, renders analytical modelling extremely challenging, if not impossible. Nevertheless modelling is manageable when suitable computational tools are used. Details of the modelling results are discussed after a discussion of the small deflection models found in the literature.

\section{$\underline{4.1 \text { Small deflection models for out-of-plane pipe whip }}$}

To date mainly small deflection, rigid-perfectly-plastic (r-p-p) pipe (beam) models have been published, which utilise extensions of classical plastic hinges. Single hinge mechanisms were analysed by Hua et al. [21]. These were extended to double hinge mechanisms [6, 11]. Triple hinge models were later developed by Reid et al. [22] and shown to be the most general mechanism required for outof-plane pipe whip. Fig. 1c shows the early stages of the deformation of a bent pipe loaded out-ofplane. A bending hinge $H_{1}$ is formed first close to the pipe end where the out-of-plane blow-down force is applied. Shortly after (see Fig. 9 below) a combined bending/torsion hinge is formed at $\mathrm{H}_{2}$ in the second segment of the pipe. In a small deflection analysis [7], both $H_{1}$ and $H_{2}$ remain stationary when the applied thrust load is constant. If the load is a rectangular pulse, when it finishes, both hinges move. $H_{1}$ will disappear before it reaches the bend and $H_{2}$ continues to travel towards the pipe root. It 
then becomes a root hinge and continues to dissipate the remaining kinetic energy under combined bending/torsion until, eventually, the pipe comes to a rest and undergoes just elastic motion.

The large deflection solution is a more difficult problem. The complexity in the required mathematical treatment for large deflections and finite rotations involving bending and torsion in 3D dimensions pose a serious challenge and question whether a pure analytical approach is warranted since alternative means such as numerical simulations are available and more manageable. Consequently a 3D nonlinear finite element model has been used herein to study the dynamic plastic motion of an out-ofplane whipping pipe.

\subsection{Large-deflection and large-rotation solution}

For simplicity, the pipe was first represented as an elastic, perfectly-plastic beam of constant cross-section. The material parameters were Young's modulus E= 200GPa, Poisson's ratio 0.3 and yield stress $\sigma_{0}=294 \mathrm{MPa}$ [19]. Fig. $1 \mathrm{~b}(\mathrm{ii})$ provides the pressure gauge readings from which the 90ms load pulse can be estimated.

Simulation can be achieved using the commercial code ABAQUS [23], through an explicit dynamic beam model. However in order to investigate the nature of local pipe collapse [24], further modelling was performed, using spatial shell elements, permitting cross-sections to deform plastically and the effects of ovalisation to be studied. A full account of the two finite element simulations is provided in [24], where it is shown that the kinematics of the pipe centreline are essentially identical. Trajectories of the pipe in 3D were obtained and the history of the pipe motion was compared with the experimental results [19] extracted from high speed films produced using the same procedure described in [9]. 
The predicted history of pipe motion obtained by the finite element modelling, compares well with the high speed film evidence of the experiments as seen in Figure 9. This verifies the reliability of nonlinear dynamic analysis by finite element for reasonably accurate determination of out-of-plane pipe whip.

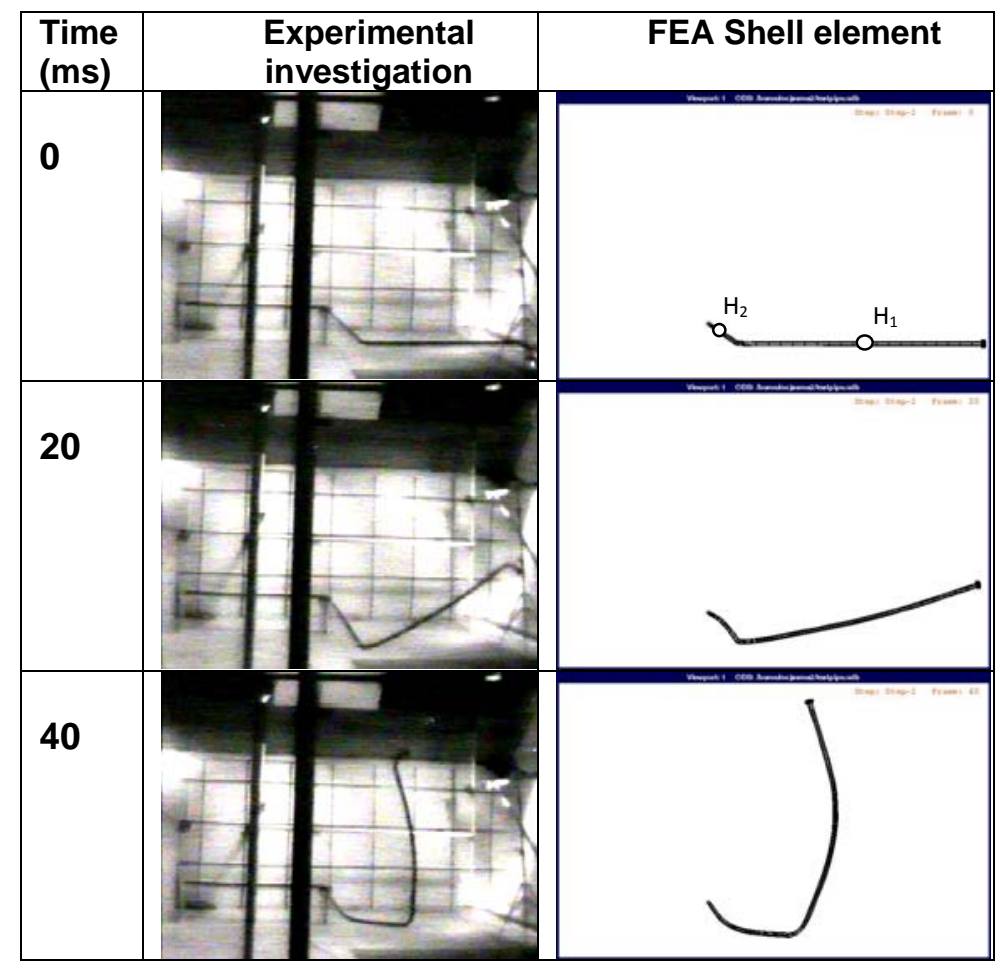




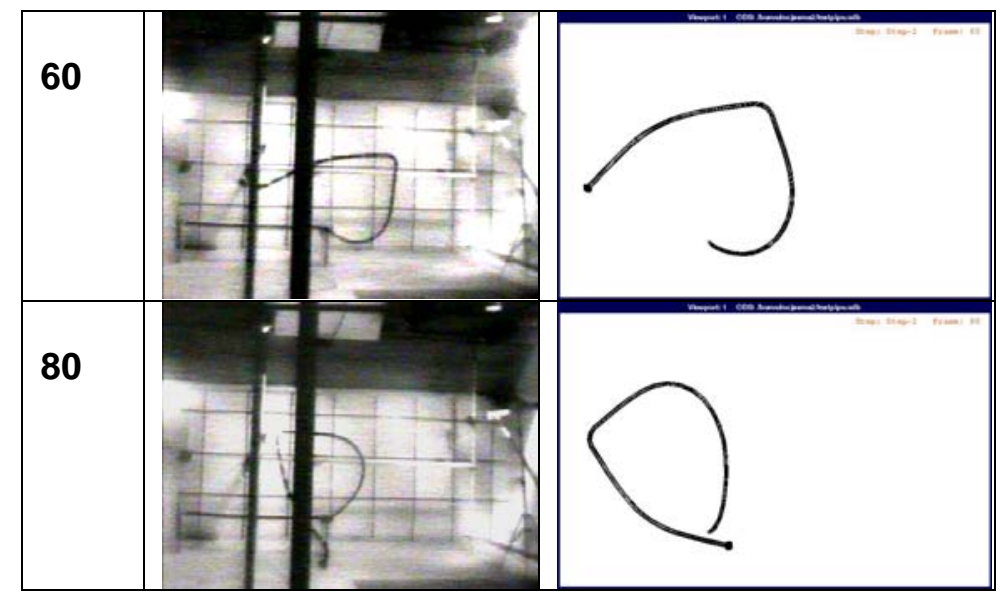

(a)

(b)

Figure 9 Out-of-plane pipe whip of a right-angled bent pipe diameter 20mm under initial pressure 1000psi.

$\mathrm{t}=0$ initial configuration; $\mathrm{t}=20-80$ ms subsequent stages in deformation. Circles at $\mathrm{t}=0$ in 9(b) indicate the initial positions of the hinge mechanism as predicted by the smalldeflection model. $\mathrm{H}_{1}$ is a pure bending hinge and $\mathrm{H}_{2}$ a combined bending-torsion hinge.

For an unconstrained three-dimensional pipe motion, the large dynamic motion of the deforming pipe resulting from the dynamic bending/torsional rotations creates a Hazard Zone (HZ). Objects within the HZ could be struck by the whipping pipe. The extent of $\mathrm{HZ}$ and the kinetic energy of the whipping pipe are clearly safety-related issues. A reasonably accurate estimate of such a potential $\mathrm{HZ}$ is of practical significance.

The dynamic, large rotation finite element model provides a means for HZ to be evaluated. Fig 10 illustrates the unconstrained dynamic motion of a right angled bent pipe under a short pulse applied at the tip A. The extent of the $\mathrm{HZ}$ is given by the coordinate limits shown as a box. This is the maximum space the unconstrained whipping pipe may strike. It is also worth noting that the excessive plastic deformation could lead to fracture in thin-walled pipes, especially at the root B. 


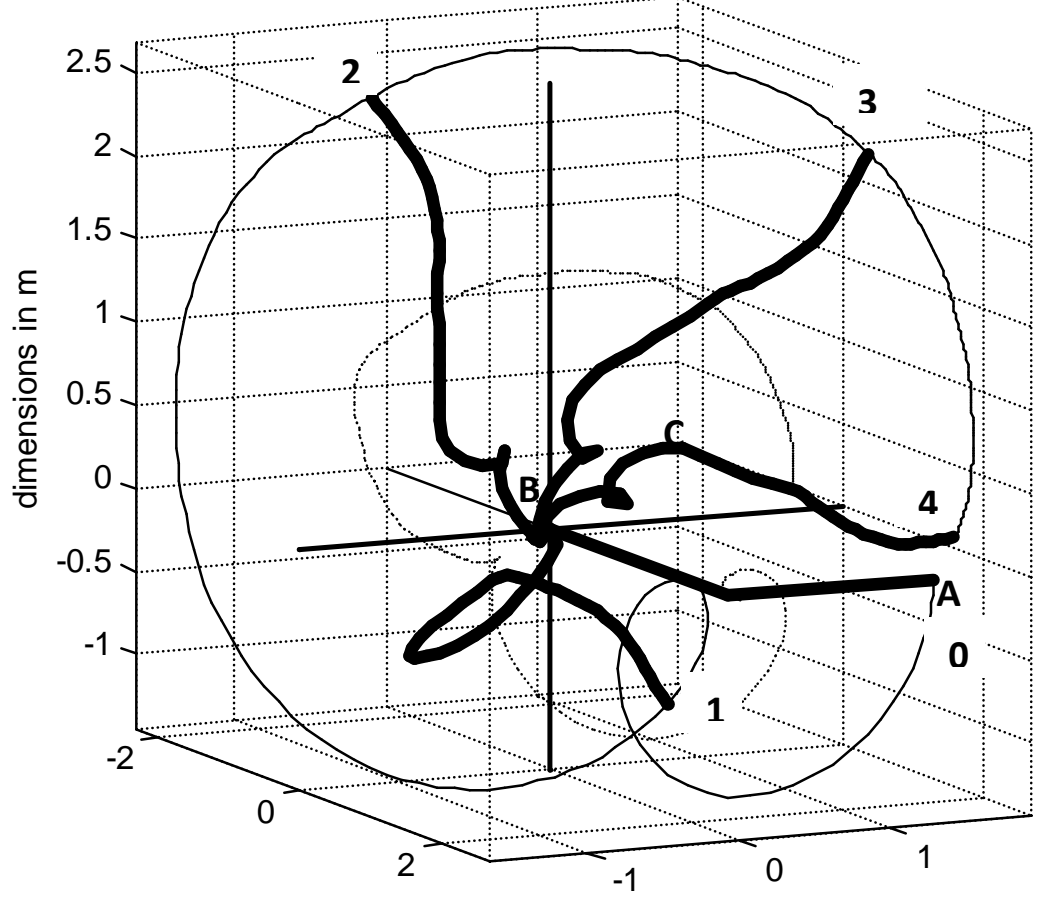

Hazard Zone (HZ) box

tip (A) and corner (C) paths 
Figure 10 Transient profile of a right-angled bent pipe under a rectangular pulse of 90ms duration. According to the model, the pipe stops after $600 \mathrm{~ms}$. Trajectories of the bent corner (C) and the pipe tip (A) are shown with dotted and lighter curves respectively. The outer box shows the Hazard Zone (space potentially affected by the dynamically deforming pipe).

Motion stages: $0(\mathrm{t}=0), 1(\mathrm{t}=89 \mathrm{~ms}), 2(\mathrm{t}=222 \mathrm{~ms}), 3(\mathrm{t}=344 \mathrm{~ms}), 4(\mathrm{t}=600 \mathrm{~ms})$

Using the sectional equivalent plastic strain [24], Fig. 11a shows first the formation and spread of the initial bending deformation which soon reaches the corner transforming into a bending-torsion zone, thereafter moving rapidly to the root. The pulse length is 90ms. Fig. 11(b) covers the rest of the motion period and shows the plastic deformation is largely concentrated at the pipe root.

(a) 

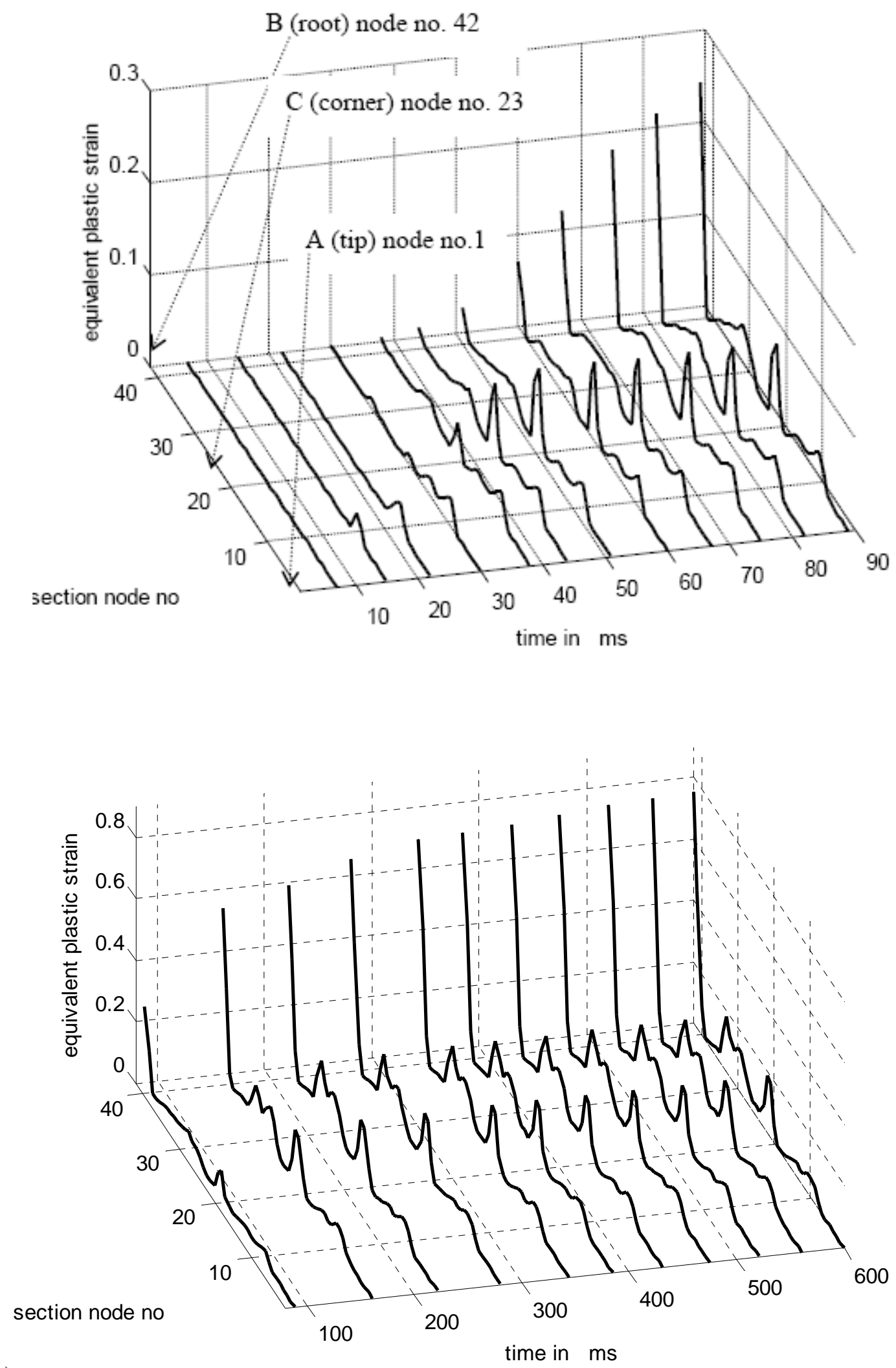

(b)

Fig. 11 
Figure 11 a: Evolution of plastic deformation in pipe cross sections during loading phase: A (tip) is node no.1 and B (root) is node no. 42, C (the corner) is node no.23. After $44 \mathrm{~ms}$ the plastic deformation switches over from $C$ to the root (B) and remains there until end

b: History of plastic deformation development in the pipe in 'modal phase. Node 1 corresponds to the pipe tip, node 23 the bent corner and 42 the root.

\section{Discussion}

The aim of this paper on the structural modelling of pipe failure analysis is directed at the specific problem of free pipe whip, the behaviour of a high pressure piping system following a potential circumferential guillotine break at a pipe section. This is a safety-related problem relevant to those industries, especially the nuclear industry, in which the potential for failure and the consequences of a break in a high pressure piping run, are key concerns. A whipping pipe could constitute a significant impact threat to neighbouring pipes and equipment.

The assessment process for such incidents provided for plant designers is currently very basic. The problem is often dealt with on an empirical or ad hoc basis. One of the most systematic and highly developed impact assessment approaches is that found in the R3 Impact Assessment Procedure produced by British Energy [2]. This is based on a compilation of international studies and simple theoretical models. As indicated in the R3 document, these models do not provide a comprehensive representation of the phenomenon of pipe whip, the designer is advised to use a full finite element representation of the event if the simpler methods provided cannot be applied. As examples of the use of FEM as the computational tool, this paper describes the work produced by the authors associated with the two generic problems of in-plane loading of right angled bent pipes and the more complex out-of-plane loading normal to the plane of the pipe. The more general case where the initial direction of the blow-down force is between these two extremes will be the subject of a future publication.

The work described in this paper is a contribution to modelling the phenomenon of pipe whip and, together with the solution of the, as yet unsolved, problem of pipe-on-pipe impact (but see [25] for a preliminary experimental and modelling investigation), could be a useful guide for designers. 


\section{Conclusions}

As shown in Sections 3 and 4, the problem of the deformation and motion of a pipe during pipe whip can be complicated. In particular, suitable computational models need to account for several significant features of the practical problem. The force applied to the end of the pipe, the blow-down force pulse, is the reaction force applied to the pipe from the (usually multi-phase) fluid jet emerging from the pipe break. Experimentally (as in the work summarised in Section 2) this is either measured or could be estimated by a separate flow calculation and depends on the 'reservoir' to which the pipe is attached [9]. Whilst pipe restraint systems could be and are installed on occasions to prevent or limit pipe whip [2], it is the prediction of the potential Hazard Zone of such a pipe and the kinetic energy that such a pipe might posses that constitute the core engineering issues.

This paper summarises some of the work that has been performed over recent years and focuses on the production of models of the structural response of a severed pipe run, but remains attached to the pressure vessel to which it is attached and hence whips.

Following a summary of the experimental work performed at UMIST in the 1990s in Section 2, the paper deals first with the modelling of a pipe that respond in a bending-dominated mode A straight cantilever pipe was considered in order to illustrate the structural complexity of the problem. Beam models (EPSH) were produced that encompass the large-deflection, plastic behaviour of a whipping pipe, idealising the pipe as an elastic-plastic beam. The ovalisation of a pipe (in the extreme the formation of a 'kink' at a section along its length) can change substantially the bending characteristics in the beam idealisation. The core of Section 3 is an exposition of the role played by elastic-plastic-hardening-softening (e-p-h-s) pipe characteristics to illustrate and discuss the numerical schemes that have been used to address the solution of this problem. The success of the model for analysing the structural response was exemplified in the comparisons of the model with high speed 
film recording of the dynamic deformation of pipes. An example of bending-only behaviour of the deformation of one- and two-dimensional bent pipe runs was provided, including straight cantilever pipes and the opening and closing modes of bent pipes loaded in their own plane. Good agreement between the model and tests demonstrate the viability of the model to represent the time-dependent deformation and kinematics of such pipes.

In Section 4 the more complex behaviour of bent pipes loaded out-of-plane was treated using the FEM code ABAQUS. The modes of deformation were described and the Hazard Zone and the details of the deformation were examined and compared with high-speed film records from the experiments. The state of stress generated in the pipe is such that the problem requires the use of an advanced computational (FEM), elastic-plastic model. Previous work [10] has demonstrated that elasticity has an important role even though the prime deformation is plastic.

In real pipe runs, the designer would ideally wish to also estimate the strain and possible failure of the whipping pipe which, in beam terms, implies the need to model the development and distribution of curvature along the length of the pipe. Whilst the e-p-h-s model was successful kinematically, the earlier central difference numerical scheme clearly exhibited oscillations that masked the true variations of the higher derivatives of the displacement and hence the curvatures. This is no doubt the case in the 3D problem, where the use of a shell model [24] adds the much-needed detail near to those sections where structural collapse can occur.

Finally, as an overview, the aim is to produce a more comprehensive and useable tool for the designers of high pressure piping system than those currently available (see [2]). With the significant and now widely available increase in computing power currently underway, such tools could be used in a Virtual Reality environment such as the one that has been used extensively in the commercial world $[1,4]$.

\section{Acknowledgement:}


The authors would like to thank the referees for their comments, especially for drawing attention to the related problem of examining the effect of the direction of the applied force as an additional parameter.

\section{References:}

[1] AVEVA PDMS 12.0, AVEVA Group plc, High Cross, Cambridge, 2010

[2] British Energy, R3 Impact Assessment Procedure, Volume 2 - Pipe Whip, 2008 (re-issue)

[3] Baum, M. R., The Rupture of High Pressure Pipe work: The Influence of Pipeline Geometry on In-Plane Pipe Whip, J. Loss Prevention and Process Industry, $\underline{9}$, No. 2, 147-159, 1996.

[4] American Nuclear Society, Time response design criteria for safety-related operator actions, ANSI/ANS-58.8, 2008

[5] Hua, Y.L., Yu, T.X. and Reid, S.R., Double-hinge modes in the dynamic response of plastic cantilever beams subjected to step loading, International Journal of Impact Engineering, Vol. 7, No. 4, pp. 401-413, 1988.

[6] Reid, S.R., Wang, B., and Yu, T.X., Yield mechanisms of a bent cantilever beam subjected to a suddenly applied constant out-of-plane tip force, International Journal of Impact Engineering, Vol. 16, No. 1, pp. 49-73, 1995.

[7] Wang, B., Yu, T.X. and Reid, S.R., Out-of-plane impact at the tip of a right-angled bent cantilever beam, Journal of Applied mechanics, Vol. 62, pp. 887-892, 1995

[8] Stronge, W.J. and Yu, T.X. Dynamic Models for Structural Plasticity, p. 80, published by Springer-Verlag, 1993.

[9] Reid, S.R., Yu, T.X., Yang, J.L. and Corbett, G.G., Dynamic elastic-plastic behaviour of whipping pipes: experiments and theoretical Model, International Journal of Impact Engineering, Vol. 18, No. 7-8, pp. 703-733, 1996.

[10] Reid, S.R., Yu, T.X., and Yang, J.L., An elastic-plastic hardening-softening cantilever beam subjected to a force pulse at its tip: a model for pipe whip, Proceedings of Royal Society London, Series A, Vol. 454, pp. 997-1029, 1998. 
[11] Reid, S.R., Hua, Y.L. and Yang, J.L., Development of a double hinge mechanisms in a bent cantilever subjected to out-of-plane force pulse, International Journal of Impact Engineering, Vol. 9, pp. 485-502, 1990.

[12] Martin, J.B. and Symonds, P.S., Mode approximation for impulsively loaded rigid-plastic structures, Journal of Engineering Mechanics, ASCE, Vol. 92, pp. 43-66, 1966.

[13] S.R. Reid, T.X. Yu and J.L. Yang, Hardening-softening behaviour of circular pipes under bending and tension, International Journal of Mechanical Sciences, Vol. 36, pp. 1073-1085, 1994.

[14] DYNA3D, Livermore Software Technology Corporation (LSTC), Livermore, California, 2000

[15] Reid, S.R. and Yang, J.L., Non-linear dynamic analysis of cantilever whipping pipe, Proceedings of Institution of Mechanical Engineers, Part E: Journal of Process Mechanical Engineering, Vol. 212, No. 3, pp. 133-149, 1998.

[16] Reid, S.R., Roy, D. and Aleyaasin, M., Pipe Whip - Recent modelling developments, Proc.. 6th International Conference on Shock \& Impact Loads on Structures, 7-9 December 2005, Perth, W. Australia, 71-82.

[17] Reid SR, Roy D. A Gaussian Sinc-Collocation approach for a Whipping Cantilever with a Follower Shear Force at its Tip., Int. J. of Num. Methods Eng 2003;58:869-92.

[18] Amit Shaw, D. Roy, S.R. Reid and M. Aleyaasin, "Reproducing kernel collocation method applied to the non-linear dynamics of pipe whip in a plane” International Journal of Impact Engineering, v 34, n 10, pp-637-1654, 2007.

[19] Wang, B. Response of two-dimensional piping system during pipe whip, Ph.D Thesis, UMIST, 1991.

[20] Reid, S. R., and Yang, J. L,.Pipe Whip: In-Plane Whipping of Bent Cantilever Pipes, Trans. ASME, J. Press. Vess Techn., 120, 70-178, 1998.

[21] Hua, Y.L. , Yu, T.X. , and Johnson, W., The plastic hinge position in a bent cantilever struck normal to its plane by a steady jet applied at its tip, International Journal of Impact Engineering, Vol. 3, No. 4, pp. 233-241, 1985.

[22] Reid, S.R., Wang, B., and Hua, Y.L., Triple plastic hinge mechanism for a bent cantilever beam subjected to an out-of-plane tip force pulse of finite duration, International Journal of Impact Engineering, Vol. 16, No. 1, pp. 75-93, 1995.

[23] ABAQUS 6.7, SIMULIA, Rising Sun Mills, Providence, 2010.

[24] Reid, S.R., Aleyaasin, M. and Wang, B. Out-of-plane pipe whip for a bent cantilever pipe: comparison between experiment and FEM models, submitted to Journal of Applied Mechanics. 
[25] Yang, J.L., Lu, G.Y., Yu, T.X. and Reid, S.R., Experimental study and numerical simulation of pipe-on-pipe impact, International Journal of Impact Engineering, Vol. 36, 1259-1268, 2009. 